\title{
High-efficiency multipass Ti:sapphire amplifiers for a continuous-wave single-mode laser
}

\author{
Patrick Georges, Frederick Estable, François Salin, ${ }^{*}$ Jean Philippe Poizat, ${ }^{\dagger}$ Philippe Grangier, and Alain Brun \\ Institut d'Optique Théorique et Appliquée, Centre National de la Recherche Scientifique, U.A. 14, Université Paris-Sud, Bâtiment 503, \\ B.P. 147, 91403 Orsay Cedex, France
}

Received May 1, 1990; accepted October 19, 1990

\begin{abstract}
We present the amplification of a continuous-wave single-mode ring dye laser in Ti:sapphire. A peak gain of $2 \times 10^{6}$ has been obtained in a passive multipass amplifier, which yielded 20 -nsec pulses of $0.7-\mathrm{mJ}$ energy at $780 \mathrm{~nm}$. We discuss the advantages of this passive multipass amplifier in comparison with a regenerative amplifier that we have also developed. By second-harmonic generation we obtained high-peak-power UV pulses from the amplified single-mode laser.
\end{abstract}

For the past few years Ti:sapphire $\left(\mathrm{Ti}: \mathrm{Al}_{2} \mathrm{O}_{3}\right)$ has been studied to develop near-infrared continuous-wave and pulsed lasers. ${ }^{1-4}$ This crystal combines the advantages of the organic dyes, which have a broad fluorescence bandwidth (650-1050 nm), and those of solidstate materials, which have a large saturation fluence $\left[1.2 \mathrm{~J} / \mathrm{cm}^{2}\right.$ (Ref. 5)]. Recently, Ti:sapphire amplifiers have been demonstrated to amplify single-frequency ${ }^{6}$ and pulsed ${ }^{7,8}$ lasers by use of the injection-seeding technique.

There is a great interest in the production of singlefrequency high-peak-power pulses for spectroscopic experiments or space-based differential absorption lidar systems. The basic idea is to produce singlelongitudinal-mode operation in a master laser, followed by a high-energy slave amplifier. Ti:sapphire is particularly well suited because of its large bandwidth and high gain.

In this Letter we report the amplification of a singlemode ring dye laser in a passive multipass configuration, which yields an output peak power of as much as $30 \mathrm{~kW}$. We have also built a regenerative amplifier, and we compare the performances and advantages of each configuration.

In our experiment the master oscillator was a krypton-ion-pumped Rhodamine 700 single-frequency ring dye oscillator tunable from 700 to $800 \mathrm{~nm}$. The linewidth of this laser is less than $1 \mathrm{MHz}$. The basic concept for the amplification in Ti:sapphire is to use the long fluorescence lifetime [ $3 \mu$ sec (Ref. 1)] to make several passes in the amplifier medium in order to obtain a high extraction efficiency.

Figure 1 is a schematic of the first amplifier that we have developed. We use four concave mirrors with slightly different radii of curvature $(1050$ and $980 \mathrm{~mm}$ ) positioned in a confocal configuration. ${ }^{9}$ These mirrors support high-reflectivity dielectric coatings for normal incidence between 600 and $900 \mathrm{~nm}$. A $25-\mathrm{mm}$ long Brewster end-cut crystal of Ti:sapphire is located at the common focal point between the mirrors. The crystal is grown using the Verneuil method by Djevahirdjian S.A. (Monthey, Switzerland). Owing to the growth process, the doping is nonhomogeneous. We used the region where the absorption is maximum, i.e., $0.48 \mathrm{~cm}^{-1}$ at $532 \mathrm{~nm}$ (corresponding to an effective absorption of $70 \%$ ). The propagation of the pulses illustrates the principle of an unstable cavity. At each pass, the beam is moving away from the optical axis of the cavity. The number of passes (six in our experiment) can be adjusted by translating a plane mirror along the $X$ axis as shown in Fig. 1. The crystal is pumped by the second harmonic of a $Q$-switched Nd:YAG laser (B.M. Industries, Evry, France) to produce 6-nsec (FWHM) pulses at a $50-\mathrm{Hz}$ repetition rate. The spatial profile of the frequency-doubled beam is Gaussian, ${ }^{5}$ while the temporal profile corresponds to that of a noninjected Q-switched Nd:YAG laser. The beam is focused by a $1-\mathrm{m}$ focal-length lens into the Ti:sapphire. Owing to the Brewster incidence in the Ti:sapphire, the beams are elliptical inside the crystal. The minimum and maximum pumpbeam diameters are 1 and $1.7 \mathrm{~mm}$, respectively, while the amplified beam diameters are 300 and $510 \mu \mathrm{m}$ (determined with a homemade beam profiler) and remain constant over all the passes.

Because of the relatively small angle of the amplified beams in the crystal, the spatial overlap is quite good along the 2.5-cm length of Ti:sapphire. Such a multipass amplifier could be designed with only two concave mirrors. However, the alignment of this amplifier is easier with four mirrors. Furthermore with the two-mirror configuration we observed a laser effect between the two facing mirrors, while this effect has been avoided with the four-mirror amplifier. The average input power of the continuous-wave beam is

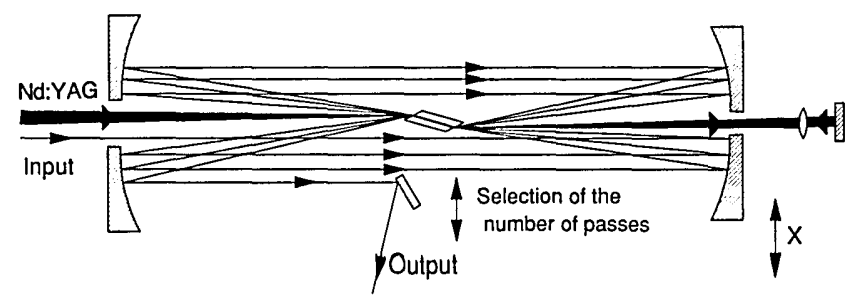

Fig. 1. Experimental setup of the multipass amplifier. 

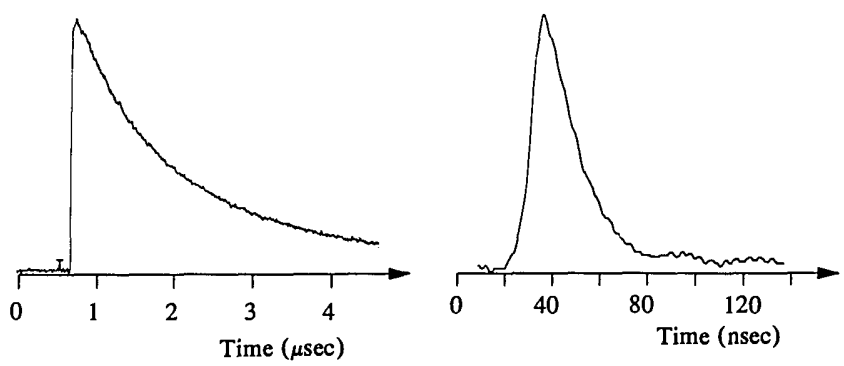

Fig. 2. Temporal profiles of the amplified pulses after one pass (left) and six passes (right; FWHM, $20 \mathrm{nsec}$ ).

$22 \mathrm{~mW}$ at $780 \mathrm{~nm}$, and the transmission of the amplifier is $80 \%$.

We measured a single-pass gain of 10 for $30-\mathrm{mJ}$ pump energy. The temporal shape of the amplified signal is determined by the fluorescence of the Ti:sapphire and has a long microsecond exponential decay. After two passes the gain is measured to be $10^{2}$, and after four passes we observe a gain of $10^{4}$.

The duration of the amplified pulses decreases with the number of passes owing to the gain sautration. After six passes we measured a pulse duration of 20 nsec (FWHM) with a short leading edge and a long trailing edge that correspond to the decrease of the gain (Fig. 2). The gain after six passes is $8 \times 10^{5}$. In order to increase the pump efficiency, we injected the transmitted pump beam back into the crystal (Fig. 1) and observed a significant increase of the amplified pulse energy. In these conditions we have obtained a gain of $2 \times 10^{6}$, and the pulse energy was $0.7 \mathrm{~mJ}$ at the output of the amplifier. ${ }^{10}$ Considering the $20-\mathrm{nsec}$ duration (FWHM), this leads to a peak power of 35 $\mathrm{kW}$ per pulse without taking into account the partially exponential tail shape. This energy corresponds to a fluence of $0.6 \mathrm{~J} / \mathrm{cm}^{2}$ in the crystal, which is close to the saturation fluence of Ti:sapphire. ${ }^{5}$ This explains the gain saturation in the crystal and the short amplified pulse duration.

The amplifier does not affect the spatial beam profile of the dye laser, and the amplified beam remains a perfect Gaussian beam. This can be explained by the larger pump-beam diameter in comparison with the amplified beam diameter. No significant amplified spontaneous emission has been observed. In fact, we did not observe any signal on a fast photodiode when we blocked the input beam. Consequently we did not need an optical isolator to preserve the dye laser from any feedback light from the amplifier, and the dye laser remained locked on a single mode.

Owing to the absence of amplified spontaneous emission and considering that the multipass amplifier does not present a longitudinal mode, the amplified pulses are expected to remain single mode. Even if the linewidth of the pulses has not been measured directly, it can be estimated to be of the order of 20 $\mathrm{MHz}$, which corresponds to the inverse of the pulse duration.

The amplified pulses are tunable from 700 to 800 $\mathrm{nm}$. This range is limited by the injection dye laser, but the gain is wavelength dependent.
To obtain high-peak-power single-mode tunable UV pulses, we used an 8-mm-thick crystal of beta barium borate (BBO) to frequency double the infrared light. Taking into account the critical phase matching of the BBO, we focused the beam with a $1-\mathrm{m}$ focal lens. The pulse energy was $18 \mu \mathrm{J}$ at $390 \mathrm{~nm}$, corresponding to a $2.5 \%$ efficiency. However, the BBO crystal used was cut for second-harmonic generation at $1.06 \mu \mathrm{m}$ for normal incidence. At $790 \mathrm{~nm}$ the phase matching is obtained by tilting the crystal. In this case the walk-off decreases the efficiency of the second-harmonic generation, and the reduced angular acceptance of $\mathrm{BBO}$ deteriorates the UV beam profile as fringes appear in the beam. This high-power singlemode source tunable in the UV ( 350 to $400 \mathrm{~nm}$ ) can find many applications in spectroscopy.

In a second experiment, we developed a regenerative amplifier ${ }^{11,12}$ in order to compare its performances with the multipass amplifier. The schematic of the regenerative cavity is shown in Fig. 3 . It is based on two highly reflective concave mirrors with 1 - and 0.5 $\mathrm{m}$ radii of curvature. The distance between the mirrors is $1.2 \mathrm{~m}$. We used the same crystal as in the previous experiment. It was pumped through mirror M1 by $6 \mathrm{~mJ}$ of energy at $532 \mathrm{~nm}$ from the $Q$-switched Nd:YAG laser. When operating in the gain-switched cavity-dumped regime (i.e., without an input beam, Pockels cells PC1 inactive, and Pockels cell PC2 used to dump the cavity), the laser produced $0.5 \mathrm{~mJ}$ of energy at $790 \mathrm{~nm}$ in an 8-nsec pulse (FWHM). This energy was limited by damages on the broadband dielectric polarizer (NRC 10FC16). The pulse duration is determined by the cavity length, and the spectrum width is approximately $20 \mathrm{~nm}$ (Fig. 4, curve a).

To injection seed the cavity, the first Pockels cell (PC1) was activated to its quarter-wave voltage, and no bias voltage was applied to the second Pockels cell (PC2). The PC2 voltage was then decreased to $0 \mathrm{~V}$ a few nanoseconds after the 532-nm pump pulse reached the Ti:sapphire crystal. At this moment a slice of the single-mode continuous-wave laser is trapped in the cavity. The duration of the slice is equal to the cavity round-trip time. To extract the amplified pulse, PC2 is activated to its quarter-wave voltage. By adjusting the time duration between the electrical pulses applied to the two Pockels cells, we control the number of passes before extraction. The pump energy is approximately $6 \mathrm{~mJ}$ on the crystal. The output from the regenerative amplifier consists of an 8-nsec (FWHM) single-mode pulse with an energy

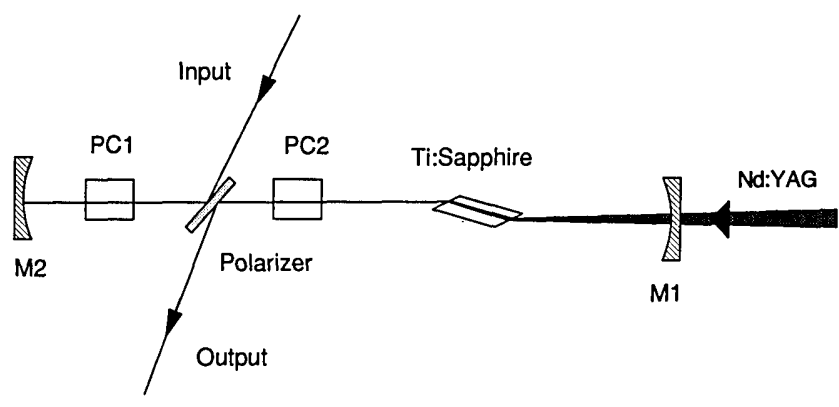

Fig. 3. Regenerative amplifier configuration. 


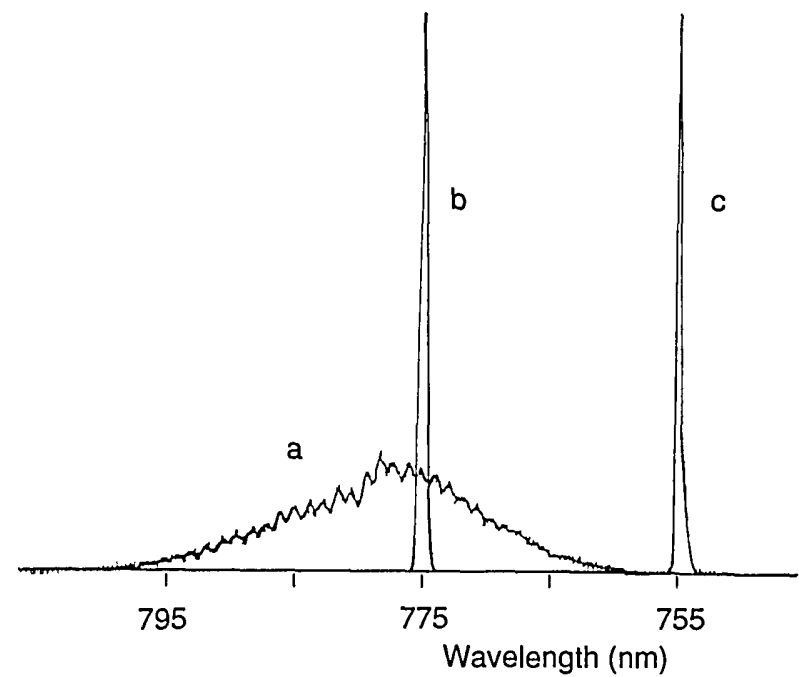

Fig. 4. Pulse spectrum after the regenerative amplifier. Curve a, the $Q$-switched regime. Curve $b$, injection seeding at $775 \mathrm{~nm}$. Curve c, injection seeding at $755 \mathrm{~nm}$. (The linewidth of the injected beams' spectrum shown here is limited by the spectrometer.)

of $0.5 \mathrm{~mJ}$ at $775 \mathrm{~nm}$. No difference in the output energy but a large decrease of the pulse buildup time is observed when the amplifier is injected seeded. The linewidth of the amplified pulses is considerably reduced (Fig. 4, curves $\mathrm{b}$ and c). We have recently shown that when a single-mode continuous-wave laser is injected in a regenerative amplifier there is no need to match the two cavity resonance frequencies and that the output of the amplifier is a single-mode pulse. ${ }^{13}$

If we assume that the pulse from this Ti:sapphire amplifier remains single mode, its linewidth is estimated to be $\sim 60 \mathrm{MHz}$. The minimum average power needed for stable injection seeding is $\sim 1 \mathrm{~mW}$. The dye laser can be tuned from 750 to $800 \mathrm{~nm}$ (limited by the mirrors' reflectivity range) while injection seeding is maintained. However, we have observed spectral filtering effects in the uncoated Pockels cells, so it is impossible to tune the dye laser continuously while maintaining the injection. The $100-\mathrm{MHz}$ modulation is attributed to a Fabry-Perot effect in the Pockels cells. This problem could be avoided by using cells with an index-matching fluid, and, in this case, highpower single-mode pulses would be produced in the near infrared.

In the regenerative amplifier, the pump efficiency is increased owing to the better mode matching between the pump and the amplified beams.

In summary, we have reported amplification with high gain in a Ti:sapphire crystal of single-mode nearinfrared emission. A passive multipass amplifier has been developed that yields 20 -nsec single-mode pulses of $0.7-\mathrm{mJ}$ energy at $780 \mathrm{~nm}$, which corresponds to a gain of $2 \times 10^{6}$. The amplifier configuration is simple and leads to high performances (in the gain, beam profile, etc.). A more complex regenerative amplifier has also been studied. The results are similar, but the single-mode dye-laser operation is easier with the multipass amplifier, whereas the pump efficiency is higher with the regenerative amplifier.

We think that this kind of amplifier configuration can be upgraded to produce higher energy and will replace the dye amplifiers in the near infrared. By using laser diodes instead of dye lasers, these all-solidstate systems could be an alternative to dye systems. Furthermore these amplifiers can be used to amplify picosecond or femtosecond pulses, ${ }^{10}$ which shows their great versatility.

The authors thank Gilles Brassart (B.M. Industries, Evry, France) for stimulating discussions, encouragements, and the loan of part of the equipment for the experiments. The research of Frederick Estable is supported by a fellowship from B.M. Industries and the Association Nationale de la Recherche Technique. At the time of these experiments Patrick Georges was also with the Ecole Polytechnique Feminine, Sceaux, France.

* Present address, Ultrafast Laboratory, University of Michigan, Ann Arbor, Michigan.

$\dagger$ Present address, Department of Physics, University of Auckland, Auckland, New Zealand.

\section{References}

1. P. F. Moulton, J. Opt. Soc. Am. B 3, 125 (1986).

2. P. Albas, E. Stark, and G. Huber, J. Opt. Soc. Am. B 3, 134 (1986).

3. J. D. Kafka, A. J. Alfrey, and T. Baer, in Ultrafast Phenomena VI, T. Yajima, K. Yoshihara, C. B. Harris, and S. Shionoya, eds., Vol. 48 of Springer Series in Chemical Physics (Springer-Verlag, Berlin, 1989), pp. 64-66.

4. J. Goodberlet, J. Wang, J. G. Fujimoto, and P. A. Schulz, Opt. Lett. 14, 1125 (1989).

5. F. Estable, F. Salin, M. Allain, P. Georges, and A. Brun, Opt. Commun. 72, 235 (1989).

6. G. A. Rines, P. F. Moulton, and J. Harrison, in Proceedings of Tunable Solid State Lasers, Vol. 5 of 1989 OSA Proceedings Series (Optical Society of America, Washington, D.C., 1989), pp. 2-8.

7. M. J. LaGasse, R. W. Schoenlein, J. G. Fujimoto, and P. A. Schulz, Opt. Lett. 14, 1347 (1989).

8. S. Basu, P. May, and J.-M. Halbout, Opt. Lett. 14, 1272 (1989).

9. E. V. Khoroshilov, I. V. Kryukov, P. G. Kryukov, and A. V. Sharkov, in Ultrafast Phenomena VI, T. Yajima, K. Yoshihara, C. B. Harris, and S. Shionoya, eds., Vol. 48 of Springer Series in Chemical Physics (Springer-Verlag, Berlin, 1989), pp. 22-23.

10. F. Estable, F. Salin, P. Georges, J. P. Poizat, and A. Brun, in Digest of Topical Meeting on Advanced SolidState Lasers (Optical Society of America, Washington, D.C., 1990), paper MC3.

11. P. Bado, M. Bouvier, and J. S. Coe, Opt. Lett. 12, 319 (1987).

12. G. Vaillancourt, T. B. Norris, J. S. Coe, P. Bado, and G. A. Mourou, Opt. Lett. 15, 317 (1990).

13. F. Salin and F. Estable, in Digest of Topical Meeting on Advanced Solid-State Lasers (Optical Society of America, Washington, D.C., 1990), paper WD6. 